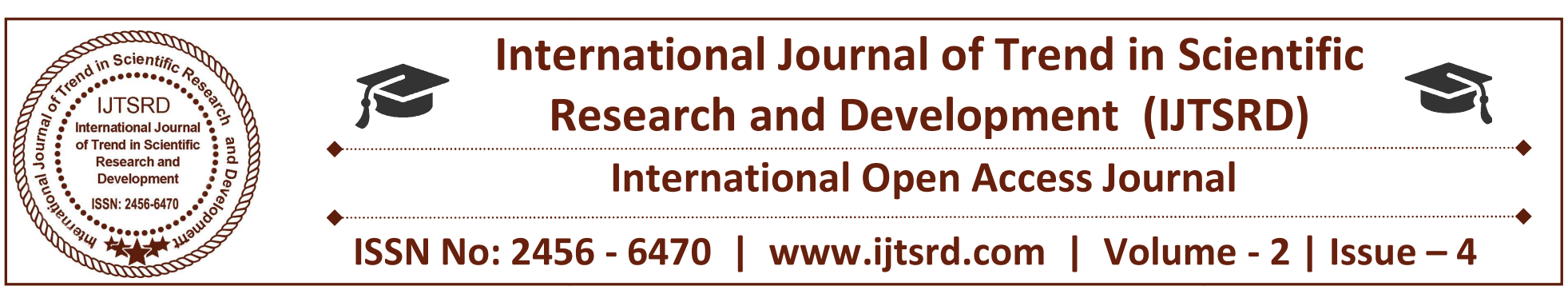

\title{
A Condition Aware Smart Navigation System
}

\author{
Ajinkya Gurav, Sneha Botalji, Rohan Lagare, Rashmi Vaidya \\ Student, Computer Engineering, Pune Vidhyarthi Griha's College of Engineering and Technology, \\ Pune, Maharashtra, India
}

\begin{abstract}
When emergencies happen, navigation services that guide people to exits while keeping them away from emergencies are critical in saving lives. To achieve timely emergency navigation, early and automatic detection of potential dangers, and quick response with safe paths to exits are the core requirements, both of which rely on continuous environment monitoring and reliable data transmission. Wireless sensor networks (WSNs) are a natural choice of the infrastructure to support emergency navigation services, given their relatively easy deployment and affordable costs, and the ability of ubiquitous sensing and communication. Although many efforts have been made to WSN-assisted emergency navigation, almost all existing works neglect to consider the hazard levels of emergencies and the evacuation capabilities of exits. Without considering such aspects, existing navigation approaches may fail to keep people farther away from emergencies of high hazard levels and would probably encounter congestions at exits with lower evacuation capabilities. In this proect, we propose a situation-aware emergency navigation system, which takes the hazard levels of emergencies and the evacuation capabilities of exits into account and provides the mobile users the safest navigation paths accordingly By guiding users following the descend gradient of the hazard potential field, System thereby can thereby achieve guaranteed success of navigation and provide optimal safety.
\end{abstract}

Keywords: WSN, Mobile communication,Microcontroller

\section{INTRODUCTION}

\section{$A$. About wireless communication in emergency} situation.
As emergency or dangerous situations change, it becomes necessary to frequently update the route plans for the guided users. Path dynamics in traditional packet routing process typically occurs between delivering different packets, while such dynamics in the navigation process might exist all along for guiding single individuals. People may even move backward to seek broader opportunities under varied situations.

There have been attempts made at guiding navigation using WSNs. Most, if not all, existing approaches assume the availability of locations on each sensor node. Knowing the locations of dangerous areas, the sensor network can perform easy and efficient route calculations to navigate internal users out of the emergency area. The location information, however, may not always be available in many realistic situations where emergency guidance are needed, for example, in an underground tunnel or coal mine, a complex indoor area, and so on. The requirement of location information largely constrains the applicability of existing approaches to location-free environments. In addition, existing approaches usually do not specifically consider the impact of variations of dangerous areas, for example, the expansion, shrinking, or disappearing of areas which is deemed dangerous. In reality, such variations often degrade the effectiveness of existing designs or even overwhelm them. So far they haven't take evacuation capability of exits into account which makes big impact in real time.

\section{$B$. Problem to be solved.}


We have arrive at the plain fact that a practical and efficient emergency navigation scheme should be situation-aware, which means that we should take into consideration both the hazard levels of concurrent emergencies and the evacuation capabilities of exits. Despite its importance, on the down side, we capitalize that it is not straightforward to design such a situation aware emergency navigation. It is nontrivial to directly extending existing methods which inherently aim at navigating users along the paths with equal distances to emergencies. The main challenge here is how to define the safety properly, incorporating the impacts of both different hazard levels of emergencies and different capabilities of the exits at the same time.

\section{Objective of project.}

The objective of a successful navigation is to plan a path for each user to one or more pre known exits on the field that lead to safe departure, bypassing all the dangerous areas. In Fig. 1, there is an exit in the fields that users are required to lead to. We depict such an example route that leads the internal user to the exit. The navigation process is carried out in a fully distributed manner without any dedicated central agents like sinks. Each user is handoff guided by the sensor along the entire route.

\section{PROPOSED WORK.}

We consider the scenario of navigating human beings on the field under emergencies, where there might be several dangerous areas that threaten the safety of human beings, People need to be guided out of the field while keeping away from those dangerous areas. Fig. 1.1 depicts such an example scenario. We characterize the navigation problem as a path planning problem and present its assumptions, objectives as follows.

Assumptions: We assume an emergent field containing several areas of dangers, as the red areas shown in Fig. 1. The dangerous areas might emerge, disappear, expand, or shrink as the time passes. The number of dangerous areas at any time is finite.

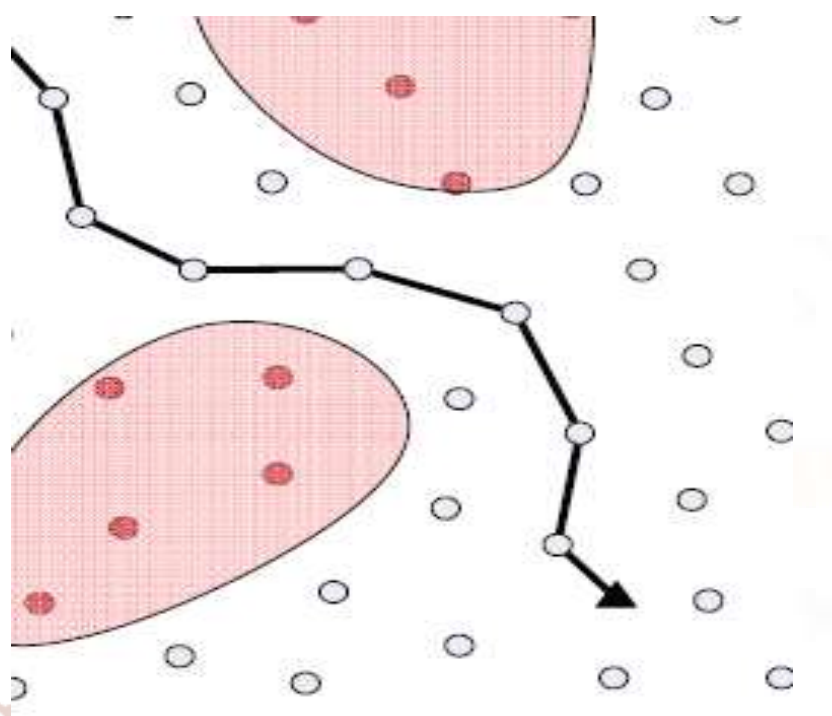

rs Alarming sensors

\section{- Internal users}

Fig. 1.1 Navigation for trapped user

A sensor network system is deployed in the field, where each sensor is able to detect the dangers distributed over the field. The sensor node triggers a "yes" alarm if it resides in the dangerous area (red nodes in Fig. 1.1) and triggers "no" if outside (skyblue nodes in Fig. 1.1). Thus, the boundary of a dangerous area can be outlined by the pairs of neighboring sensors with different outcomes. Each user carries a communicating device that can talk with system. By measuring the strength and direction of wireless signals, the user is able to track any targeted sensor node. Thus, the navigating route can be interpreted as a sequence of nodes.

\section{Related work:}

A variety of research works have been done on these issues ranging from utilization of GPS systems to using signal strengths from nodes and coordinators. GPS-based techniques are one of the commonly used techniques in WSNs and are mostly used in largescale WSNs [2], [3]. However, GPS based systems suffer from drawbacks, like high cost (at times more than the cost of a small sensor node), inaccuracy in working when in an indoor environment and very high energy-consumption [4] and are thus not suitable for small scale WSNs. [6]-[8] propose variety of range-free and range based target tracking and localization algorithms in WSNs.

While, range-free algorithms calculate a sensor nodes location from the network connectivity [6], the range- 
based algorithms mostly rely on anchor nodes to supply the distance measurements and then perform triangulation or maximal likelihood estimation techniques to calculate the locations of unknown nodes in a WSN. The commonly known techniques to calculate the range information include measuring the Time of Arrival (TOA), Time Difference of Arrival (TDOA), Received Signal Strength Indicator (RSSI) [7] etc.

The schemes proposed in our work use RSSI to perform the target tracking and localization techniques. Another commonly used parameter for location tracking and localization in WSNs is the received signal strengths (RSS) from coordinators and sensor nodes [4]. RSS (in its different forms) may form the basis for determining the requirement of a handover activity and tracking/deciding on the target coordinator to handover with. Commonly used parameters in this regard are the Received Signal Strength Indicator (RSSI) [1], the Link Quality Indicator (LQI) [2], and the Packet Reception Rate (PRR) [3]. However, as considering only RSSI-based or LQI based measurements for target tracking during a handover may result in inappropriate decision and selection of the handover target, other essential parameters to consider, in this context, are movement direction, speed and distance of the device [4]. We propose here three different target tracking schemes, primarily based on RSSI, during handover activities in WSNs. Other parameters considered for the schemes are distance and movement direction of the sensor nodes.

\section{CONCLUSION:}

This system conducts the first work on situationaware emergency navigation by considering a more general and practical problem, where emergencies of different hazard levels and exits with different evacuation capabilities may coexist. We first model the situation-aware emergency navigation problem and formally define the safety of a navigation path.

We are currently devoting to conducting a small-scale system prototype under more complex scenarios. In the future, we would like to explore modeling the hazard speed in the context of emergency navigation. We also plan to cooperate with the local Fire Department to test our prototype, e.g., in the fire fighting exercises, to provide more evidences on the real effects on user safety in real scenarios.
We are the authors from the University of Pune wish to acknowledge the contribution of all support staff of Pune Vidyarthi Griha's College of Enginering(Computer Department) who have helped us for the success of this research.

\section{REFERENCES}

1) Amundson and X. D. Koutsoukos, "A survey on localization for mobile wireless sensor networks," in Mobile Entity Localization and Tracking in GPS-less Environnments. Springer, 2009, pp. 235-254.

2) T. He, P. Vicaire, T. Yan, L. Luo, L. Gu, G. Zhou, R. Stoleru, Q. Cao, J. A. Stankovic, and T. Abdelzaher, "Achieving real-time target tracking using wireless sensor networks," in Real-Time and Embedded Technology and Applications Symposium, 2006. Proceedings of the $12^{\text {th }}$ IEEE. IEEE, 2006, pp. 37-48.

3) A. Brown, J. Griesbach, B. Bockius, and T. Boult, "Gps tracking location-based service using wristwatch geozigbee sensors," in Proceedings of the ION National Technical Meeting, 2007.

4) D. Capriglione, L. Ferrigno, E. D’Orazio, V. Paciello, and A. Pietrosanto, "Reliability analysis of rssi for localization in small scale wsns," in Instrumentation and Measurement Technology Conference (I2MTC), 2012 IEEE International. IEEE, 2012, pp. 935-940.

5) "S. Ray, J. Kumar, S. Sen, and J. Nath, "Modified distance vector routing scheme for a manet," in The National Conference on Communications, Kanpur, India, 2007, pp. 197-202.

6) D. Niculescu and B. Nath, "Dv based positioning in ad hoc networks," Telecommunication Systems, vol. 22, no. 1-4, pp. 267-280, 2003.

7) M. L. Sichitiu and V. Ramadurai, "Localization of wireless sensor networks with a mobile beacon," in Mobile Ad-hoc and Sensor Systems, 2004 IEEE International Conference on. IEEE, 2004, pp. 174-183.

8) R. Priwgharm and P. Chemtanomwong, "A comparative study on indoor localization based on rssi measurement in wireless sensor network," in Computer Science and Software Engineering (JCSSE), 2011 Eighth International Joint Conference on. IEEE, 2011, pp. 1-6.

\section{ACKNOWLEDGEMENT:}


9) A. H. Sayed, A. Tarighat, and N. Khajehnouri, "Network-based wireless location: challenges faced in developing techniques for accurate wireless location information," Signal Processing Magazine, IEEE, vol. 22, no. 4, pp. 24-40, 2005.

10) G. Sun, J. Chen, W. Guo, and K. R. Liu, "Signal processing techniques in network-aided positioning: a survey of state-of-the-art positioning designs," Signal Processing Magazine, IEEE, vol. 22, no. 4, pp. 12-23, 2005.

11) S. Kim, S. Kim, and D.-S. Eom, "Rssi/lqi-based transmission power control for body area networks in healthcare environment," Biomedical and Health Informatics, IEEE Journal of, vol. 17, no. 3, pp. 561-571, 2013.
12) N. Baccour, A. Koub`aa, L. Mottola, M. A. Z'úniga, H. Youssef, C. A. Boano, and M. Alves, "Radio link quality estimation in wireless sensor networks: A survey," ACM Trans. Sen. Netw., vol. 8, no. 4, pp. 34:1-34:33, Sep. 2012. [Online]. Available:

http://doi.acm.org/10.1145/2240116.2240123

13) M. Rondinone, J. Ansari, J. Riihij ${ }^{a r v i, ~ a n d ~ P . ~}$ M"ah"onen, "Designing a reliable and stable link quality metric for wireless sensor networks," in Proceedings of the workshop on Real-world wireless sensor networks, ser. REALWSN '08. New York, NY, USA: ACM, 2008, pp. 6-10. 